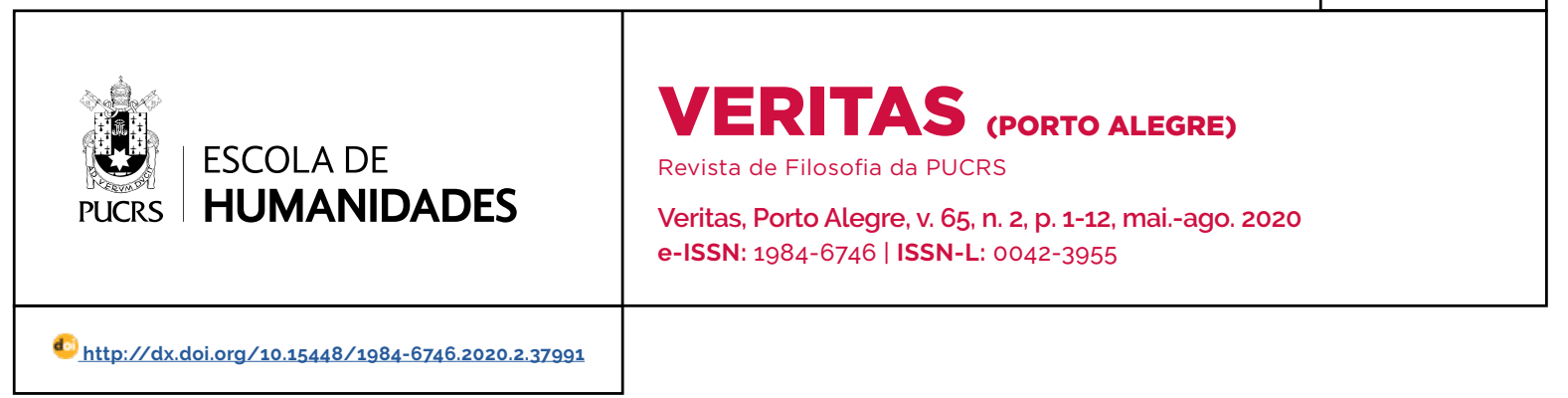

SEÇÃO: VARIA

\title{
Bioética em tempos de pandemia: Testes clínicos com Cloroquina para tratamento de COVID-19 ${ }^{1}$
}

\author{
Bioethics in times of pandemic: Clinical trials with Chloroquine for the treatment of COVID-19 \\ Bioética en tiempos de pandemia: Ensayos clínicos con cloroquina para el tratamiento \\ de COVID-19
}

\section{Alcino Eduardo Bonella $^{2}$ orcid.org/0000-0002-7910-6961 abonella@ufu.br}

\author{
Marcelo de Araujo 3 \\ orcid.org/0000-0001-8934-6195 \\ marcel@pobox.com
}

\section{Darlei Dall'Agnol 4}

orcid.org/0000-0003-4203-1094 ddarlei@yahoo.com

Recebido em: 5 mai. 2020 Aprovado em: 20 mai. 2020. Publicado em: 28 jul. 2020.

\section{(c) (1)}

Artigo está licenciado sob forma de uma licença Creative Commons Atribuição 4.0 Internacional.
Resumo: Defendemos a necessidade de que se atue com rigor ético durante a pandemia do novo coronavirus e que não se afrouxem os padrões científicos e normativos nas pesquisas sobre possiveis medicamentos, em especial a Cloroquina, para a COVID-19. Argumentamos que, excepcionalmente, seria justificado pular algumas etapas na realização de testes clínicos ou tratamentos experimentais, mas que é inadequado fazer um uso indiscriminado de medicamentos off label para tratar pacientes enquanto os resultados das pesquisas não forem publicados em veículos de comprovada reputação científica. Para sustentar essa posição, aplicamos principios bioéticos e da ética médica. Defendemos que políticas públicas para o uso de Cloroquina durante a pandemia não devem ser implementadas até que sejam divulgados os resultados das pesquisas realizadas pela "Coalizão COVID Brasil" e pela Solidarity da Organização Mundial da Saúde (OMS).

Palavras-chave: Bioética. COVID-19. Pandemia. Ética em pesquisa. Novos medicamentos. Políticas públicas na área da saúde.

Abstract: We defend the need to act with ethical rigor during the pandemic of the novel coronavirus and not to loosen scientific and normative standards in research on possible drugs, especially chloroquine, for COVID-19. We argue that, exceptionally, it would be justified to skip some steps in conducting clinical trials or experimental treatments, but that it is inappropriate to make indiscriminate use of off-label drugs to treat patients until the results of the research are published in vehicles of proven scientific reputation. To support this position, we apply bioethical principles and medical ethics. We argue that public policies for the use of Chloroquine during the pandemic should not be implemented until the results of the research carried out by the «COVID Brasil Coalition" and by WHO (World Health Organization) Solidarity are released.

Keywords: Bioethics. COVID-19. Pandemic. Research ethics. New drugs. Public health policies.

Resumen: Defendemos la necesidad de actuar con rigor ético durante la pandemia del nuevo coronavirus y no de flexibilizar las normas cientificas y normativas en la investigación sobre posibles fármacos, especialmente la cloroquina, para COVID-19. Argumentamos que, excepcionalmente, estaría justificado omitir algunos pasos en la realización de ensayos clinicos o tratamientos experimentales, pero que no es apropiado hacer un uso indiscriminado de medicamentos off label para tratar a los pacientes hasta que los resultados de la investigación se publiquen en vehículos de probada reputación científica. Para mantener

\footnotetext{
O presente artigo resultou do trabalho de um grupo de pesquisadores, organizado pelo Prof. Dr. Marco Antônio Azevedo (UNISINOS) chamado "Dilemas COVID-19 Bioética," a quem os autores agradecem a leitura atenta, bem como as suas críticas e contribuições. Os autores agradecem também ao CNPq, pelo financiamento de suas respectivas pesquisas.

2 Universidade Federal de Uberlândia (UFU), Uberlândia, MS, Brasil.

3 Universidade do Estado do Rio de Janeiro (UERJ), Rio de Janeiro, RJ, Brasil. Universidade Federal do Rio de Janeiro (UFRJ), Rio de Janeiro, RJ, Brasil.

4 Universidade Federal de Santa Catarina (UFSC), Florianópolis, SC, Brasil.
} 
esta posición, aplicamos principios bioéticos y ética médica. Argumentamos que las políticas públicas para el uso de cloroquina durante la pandemia no deben aplicarse hasta que se publiquen los resultados de la investigación realizada por la "Coalición COVID Brasil" y la Organización Mundial de la Salud (OMS Solidaridad) Palabras clave: Bioética. COVID-19. Pandemia. Ética de la investigación. Nuevos medicamentos. Políticas públicas en el área de la salud.

\section{Introdução}

Para que a pandemia de COVID-19 (doença causada por vírus da espécie SARS-Cov-2, gênero betacoronavirus, da família coronavirus) não assuma proporções catastróficas no Brasil, devemos atentar para a eticidade das ações dos diversos atores envolvidos na geração de políticas públicas, incluindo as ações de médicos e pesquisadores responsáveis pelas pesquisas científicas com seres humanos acometidos pela COVID-19.

Um domínio no qual a Bioética pode nos guiar diz respeito à reflexão crítica no desenvolvimento dos mecanismos necessários para combater a COVID-19, tais como, por exemplo, produtos diagnósticos, reposicionamento de drogas (drug repositioning em inglês), e criação de vacinas específicas. Dada a urgência da situação, seria tentador, durante a pandemia, usar indiscriminadamente remédios off label, em caráter "experimental", sem a devida testagem clínica para comprovar a sua eficácia e segurança. E de fato é isso que vem ocorrendo no Brasil no que concerne ao uso da Cloroquina (doravante CLQ) para o tratamento da COVID-19. Mas como pretendemos mostrar no presente artigo, devemos resistir a essa tentação e observarmos alguns principios fundamentais da Bioética.

Nossa hipótese é a de que, excepcionalmente, durante uma pandemia, seria eticamente aceitável pular algumas das fases previstas nos protocolos internacionais para testes clínicos de novos medicamentos. Isso significa dizer que, para o benefício da pesquisa, alguns pacientes poderiam participar voluntariamente, e com o devido consentimento esclarecido, de pesquisas experimentais que seguirão um protocolo diferente das pesquisas são geralmente feitas fora do periodo de pandemia. No entanto, o tratamento de pacientes que não fazem parte dessas pesquisas experimentais não pode ser feito antes da publicação dos resultados das pesquisas.

Quaisquer que sejam as decisões tomadas, precisamos discuti-las publicamente, com transparência e boa-fé, colocando o bem-estar dos pacientes acima de qualquer outro interesse. Em todos os casos, precisamos confiar nos profissionais de saúde e nos cientistas para que menos injustiças sejam cometidas e mais vidas sejam salvas.

\section{Testes clínicos e uso off label de medicamentos}

O uso de medicamentos em caráter experimental para tratar a COVID-19 começou a ser feito no Brasil sem a devida aprovação dos órgãos governamentais competentes e, mais tarde, com testes clínicos com previsão de pular etapas. Nesta primeira seção, reconstruiremos o debate atual, tanto científico quanto ético, em torno do uso da Hidroxicloroquina (doravante HCLQ), menos tóxica que a CLQ, combinado ou não à Azitromicina, para tratamento da COVID-19.

Para entender a relevância do tema, basta lembrar que as cinco primeiras mortes pela COVID-19 no Brasil ocorreram no município de São Paulo, em pacientes internados no Hospital Sancta Maggiori, da rede Prevent Senior, que estaria sendo inclusive investigado pela Prefeitura por não ter informado às autoridades competentes sobre a admissão de pessoas contaminadas pelo novo coronavírus. ${ }^{2}$ Em 22 de março de 2020, foi divulgado que a mãe dos sócios-fundadores da Prevent Senior, uma senhora de 75 anos, teria sido a primeira a ser tratada com CLQ e Azitromicina. ${ }^{3}$ Trata-se de um uso off label que ainda não fora autorizado, pois tais medicamentos foram desenvolvidos e testados originalmente para

\footnotetext{
MOREIRA; WATANABE, P.; SALDAÑA, P. Prevent Senior, em SP, tem cinco mortes por novo coronavirus; Brasil soma sete. Folha de S.Paulo, 19 mar. 2020. Disponivel em: https://www1.folha.uol.com.br/equilibrioesaude/2020/03/prevent-senior-tem-cinco-mortes-por-novo-coronavirus-em-sp-brasil-soma-sete.shtml. Acesso em: 25 mar. 2020.

3 A administração das drogas teria sido feita entre os dias 17 e 18 de março de 2020, conforme entrevista publicada na Folha de S.Paulo GRANCHI, G. Entrevista com Prevent Senior. Viva Bem, São Paulo, 22 mar. 2020. Disponivel em: ttps://noticias.uol.com.br/saude/ultimas-noticias/redacao/2020/03/23/prevent-senior-usa-cloroquina-em-todos-os-pacientes-com-coronavirus.htm. Acesso em: 30 mar. 2020.
} 
outras doenças tais como, por exemplo, malária e lúpus. Naquele momento, não foi informado se outros pacientes com COVID-19 também estavam sendo tratados com o mesmo medicamento. Foi apenas um dia depois que a Prevent Senior anunciou publicamente que todos os seus pacientes que testaram positivo para o COVID-19 estavam sendo tratados com CLQ. ${ }^{4}$ No entanto, não havia autorização governamental para isso, e também não existiam evidências científicas suficientes acerca da segurança e eficácia do desse tipo de tratamento.

Naquele momento, a CLQ se popularizou a partir de declarações, nas diversas mídias sociais, dos presidentes americano Donald Trump e brasileiro Jair Bolsonaro. Muitas pessoas correram às farmácias e esgotaram os estoques, deixando pessoas que sofrem de malária e lúpus em situação ainda mais vulnerável e com medo de que faltasse o medicamento originalmente produzido para tratá-las. Novamente, poucas evidências existiam e a promessa de cura era precipitada. Esse é exatamente o comportamento irresponsável que precisamos evitar em tempos de pandemia. Além disso, tais substâncias não têm efeito preventivo e podem, como já se sabe, até mesmo intoxicar algumas pessoas. ${ }^{5}$ Ao invés de curá-las, essas drogas podem causar, entre outros, problemas cardiovasculares e levá-las a óbito como, já ocorreu vários países. ${ }^{6}$

No mesmo dia em que o presidente brasileiro defendia o uso da CLQ em rede nacional de televisão (08/04/20), a revista científica The British Medical Journal apontava para os seus efeitos adversos e alertava que seu uso generalizado como tratamento para a COVID-19 era prematuro:
O uso ampliado da Hidroxicloroquina expõe alguns pacientes a danos, potencialmente fatais, incluindo reações adversas cutâneas graves, insuficiência hepática fulminante e arritmias ventriculares (principalmente quando prescritas com Azitromicina). ${ }^{7}$

Se esse é o caso, então nada mais razoável, tanto científica quanto eticamente, do que usar principios bioéticos, da ética médica e até mesmo o princípio de precaução para evitar danos maiores. Esse ponto será desenvolvido mais adiante. Seja como for, não nos parece moralmente adequado tratar pessoas sem o embasamento de pesquisas científicas, desenvolvidas de modo eticamente aceitável, ou usar medicamentos off label em caráter experimental, sem o devido acompanhamento médico.

Não é claro qual seria o embasamento para a escolha do tratamento com CLQ na pandemia de COVID-19. De fato, algumas pesquisas estavam sendo feitas, mas elas não eram conclusivas. Um estudo mencionado foi o liderado pelo pesquisador Philippe Gautret. ${ }^{8}$ Esse estudo, ainda que limitado, parecia animador para a combinação de HCLQ e azitromicina, mas ele também recebeu várias críticas. Além da pequena amostragem, uma das críticas levantou dúvidas quanto a metodologia empregada. Afinal, tratava-se de um ensaio clínico aberto, não randomizado, um tipo de estudo sujeito a vieses de confusão. Além disso, parte do grupo controle foi composto por pacientes que se recusaram a receber o tratamento. Estudos desse tipo levantam muitas dúvidas: como assegurar-se de que o resultado positivo alegadamente observado, de uma ação sinérgica entre a azitromicina e a HCLQ na COVID-19 não foi fruto do acaso? A falta de randomização na seleção dos grupos de tratamento e

\footnotetext{
4 Prevent Senior usa cloroquina em todos os casos com covid-19, diz diretor. Folha de S.Paulo, São Paulo, 23 mar. 2020. Disponível em: https://noticias.uol.com.br/saude/ultimas-noticias/redacao/2020/03/23/prevent-senior-usa-cloroquina-em-todos-os-pacientes-com-coronavirus.htm. Acesso em: 30 mar. 2020.

5 MAKIN AJ. et al. Fulminant hepatic failure secondary to hydroxychloroquine. Gut, n. 35, p. 569-570, 1994. Disponivel em: https://wwww. ncbi.nlm.nih.gov/pmc/articles/PMC1374814/pdf/guto0538-0147.pdf. Acesso em: 30 maio 2020

6 CHATRE C. et al. Cardiac Complications Attributed to Chloroquine and Hydroxychloroquine: A Systematic Review of the Literature. Drug Saf. 2018 Oct, v. 41, n. 10, p. 919-931. Disponivel em: https://www.ncbi.nlm.nih.gov/pubmed/29858838. Acesso em: 7 maio 2020.

7 FERNER, R.; ARONSOR, J. Chloroquine and hydroxychloroquine in covid-19. Use of these drugs is premature and potentially harmful Editorials. BMJ 2020; 369:m1432 doi: 10.1136/bmj.m1432 Disponivel em: https://www.bmj.com/content/bmj/369/bmj.m1432.full.pdf. Acesso em: 9 abr. 2020.

8 GAUTRET, P., et al. Hydroxychloroquine and azithromycin as a treatment of COVID-19: results of an open-label non-randomized clinical trial. International Journal of Antimicrobial Agents. 20 mar. 2020. DOI: https://doi.org/10.1016/j.ijantimicag.2020.105949 Disponivel em: https://www.ncbi.nlm.nih.gov/pubmed/32205204. Acesso em: 26 mar. 2020.
} 
controle é certamente uma falha metodológica que lança muitas dúvidas sobre as conclusões apresentadas no estudo. Reconhecer esses limites metodológicos é fundamental para que não generalizemos apressadamente os resultados do estudo, por mais promissores que pareçam.

A pesquisa pode, então, ser criticamente questionada e precisa ser contrastada com outras investigações científicas e testes clínicos similares. De fato, um trabalho publicado no Journal of Zhe Jiang University, relatando pesquisa liderada por Jun Chen, já apresentava a conclusão de que não haveria diferença significativa entre o grupo tratado com HCLQ e Azitromicina e o não tratado. ${ }^{9}$ Os pesquisadores sugerem nas conclusões, de forma cautelosa, que novas pesquisas sejam realizadas. O hospital francês Pitié-Salpêtrière também testou a HCLQ e concluiu que ela não se mostrara eficaz no tratamento da COVID-19. ${ }^{10}$ Um estudo similar, feito pela Fiocruz e pela Fundação de Medicina Tropical, no Brasil, sugere que a letalidade de pacientes com COVID-19 tratados com CLQ é praticamente a mesma dos não tratados. ${ }^{11}$ Novamente, antes de recomendar o uso da CLQ em políticas públicas para o combate à pandemia, líderes políticos deveriam ter aguardado a realização de mais testes clínicos. Naquele momento, as evidências científicas de beneficio eram fracas e, portanto, insuficientes para indicações médicas inequivocas de uso da HCLQ para tratamento da COVID-19.

Não demorou muito, então, para que a comunidade cientifica internacional propusesse a realização de mais pesquisas clínicas a fim de avaliar a eficácia da CLQ no tratamento da COVID-19. Pouco tempo depois, então, alguns hospitais brasileiros de credibilidade anunciaram a formação de uma coalização para iniciar testes com medicamentos que poderiam aliviar os sintomas e ajudar na recuperação da COVID-19. A descrição da pesquisa foi feita nestes termos por um dos participantes dessa coalizão:

Uma parceria entre o Hospital Israelita Albert Einstein, HCor, Hospital Sírio Libanês, Hospital Moinhos de Vento, Hospital Alemão Oswaldo Cruz, BP - A Beneficência Portuguesa de São Paulo e Rede Brasileira de Pesquisa em Terapia Intensiva (BRICNet), junto com o Ministério da Saúde, inicia pesquisas para avaliar a eficácia e segurança de medicamentos para pacientes com infecção pelo novo coronavirus (COVID-19). Com o apoio na pesquisa da farmacêutica EMS fornecendo os medicamentos Hidroxicloroquina e Azitromicina. Participarão do estudo entre 40 e 60 hospitais.

A iniciativa foi nomeada de Coalizão COVID Brasil, e contará com 40 a 60 hospitais do Pais para realizar três pesquisas. Coalizão I, a primeira pesquisa, envolverá pacientes de menor gravidade internados por COVID-19. Nestes pacientes será avaliado se a Hidroxicloroquina é eficaz em melhorar o quadro respiratório. Também será avaliado se adicionar o medicamento Azitromicina, que pode potencializar a ação da Hidroxicloroquina, terá efeito benéfico adicional. Serão incluidos nesta primeira fase 630 pacientes.

A Coalizão Il, segunda pesquisa, envolverá casos mais graves, que necessitam de maior suporte respiratório. Nesta, todos os pacientes receberão o medicamento Hidroxicloroquina, com o objetivo de verificar se a Azitromicina tem efeito benéfico adicional, com potencial de melhorar os problemas respiratórios causados pelo novo coronavirus. Os centros participantes são os mesmos e serão incluidos em torno de 440 pacientes.

O terceiro estudo, Coalizão III, avaliará a efetividade da dexametasona, uma medicação com ação anti-inflamatória, para pacientes com insuficiência respiratória grave, que necessitam de suporte de aparelhos (ventilador mecânico) para respirar. Nesta pesquisa serão incluidos 284 pacientes..12

Essa atitude é claramente mais adequada, tanto cientificamente quanto eticamente, pois são necessárias pesquisas mais amplas para se estabelecer a eficácia e a segurança desses medicamentos, quando usados para o tratamento de COVID-19. Enquanto não aparecerem mais evidências em favor do uso desses medicamentos

\footnotetext{
$9 \mathrm{CHEN}$, J. et al. A pilot study of hydroxychloroquine in treatment of patients with common coronavirus disease-19 (COVID-19). Journal of Zhe Jiang University, v. 49, n. 1, Hangzhou, 2020. DOI: 10.3785/j.issn.1008-9292.2020.03.03. Disponivel em: http://www.zjujournals.com/ med/EN/10.3785/j.issn.1008-9292.2020.03.03. Acesso: 30 mar. 2020.

10 FOURCADE, M. Coronavírus: hidroxicloroquina não mostra eficácia em hospital francês. São Paulo, Folha de São Paulo. Disponível em: https://noticias.uol.com.br/ultimas-noticias/bloomberg/2020/04/02/coronavirus-hidroxicloroquina-nao-mostra-eficacia-em-hospital-frances.htm. Acesso em: 7 abr. 2020.

11 Medicamentos como hidroxicloroquina e cloroquina funcionam contra o coronavirus? Rio de Janeiro, Fiocruz. Disponivel em: https:// portal.fiocruz.br/pergunta/medicamentos-como-hidroxicloroquina-e-cloroquina-funcionam-contra-o-coronavirus. Acesso em: 7 abr. 2020 12 "Coalizão COVID Brasil" convida médicos, pesquisadores e profissionais da saúde para pesquisa sobre tratamento de Coronavírus. Disponivel em: https://www.hcor.com.br/imprensa/noticias/coalizao-covid-brasil-convida-medicos-pesquisadores-e-profissionais-da-saude-para-pesquisa-sobre-tratamento-de-coronavirus/. Acesso em: 17 abr. 2020.
} 
durante a pandemia de COVID-19, as indicações podem ser apenas opcionais, ou seja, os benefícios não são comprovados e a avaliação clínica deve ser feita caso a caso pelo profissional da saúde. Todavia, como os resultados somente sairão em 60 ou 90 dias nada pode ser concluído sobre uso em larga escala no presente momento (30/4/20). Seja como for, a aprovação pela Comissão Nacional de Ética em Pesquisa, (CONEP), em 20/3/2020, e pela Agência Nacional de Vigilância Sanitária (ANVISA), em 27/3/2020, foi feita em regime de urgência. Seria prudente, por conseguinte, esperar o resultado das pesquisas da Coalizão COVID Brasil e, principalmente, da Organização Mundial de Saúde (OMS) para não extrapolar indevidamente os usos da CLQ ou da HCLQ.

No entanto, já em 25/3/2020, o Ministério da Saúde recomendou o uso off label da HCLQ para tratamento dos casos mais graves e críticos da COVID-19. Em um comunicado técnico (Nota 5), o Ministério da Saúde indicou também o protocolo e posologia que deveriam ser observados no tratamento. ${ }^{13} \mathrm{~A}$ dosagem autorizada é de 3 comprimidos de 150g/dia, ou seja, no máximo, 450g. A diretriz afirmava que era uma terapia adjuvante, e não falava de cura para a COVID-19. Entretanto, como veremos mais adiante, o seu uso passou a ser feito nas fases iniciais da doença justificado, muitas vezes, com explicações duvidosas como, por exemplo, a de que a HCLQ reduziria o tempo de internação, a mortalidade etc. Tais usos não possuíam embasamento nem científico nem bioético e deveriam, portanto, ser repudiadas. Dito de outro modo, não se seguiu a recomendação das autoridades nacionais responsáveis nem se esperou pela divulgação dos resultados dos testes clínicos. Os poucos "estudos" empíricos, baseados apenas em narrativas e relatos pessoais de poucos casos, não observavam o padrão de testes clínicos rigorosos. Tais atitudes afrontam os principios que guiam os padrões atuais da boa medicina baseada em evidências (MBE) e desrespeitam os princípios bioéticos.

\section{Razões bioéticas para testagem em regime de urgência}

Nesta seção, discutiremos a permissibilidade ou não de se pular etapas nos ensaios clínicos para novos medicamentos ou do uso off label de remédios produzidos para outras enfermidades. Em condições normais, como veremos, para a produção de medicamentos novos, há um processo de testagem clínica que vai de um pequeno número de pessoas sadias para avaliar os riscos, passando por uma amostragem de pessoas contaminadas para avaliar a eficácia, até o uso controlado em larga escala. Seria correto pular alguma dessas etapas durante uma pandemia? Ou seria moralmente justificado usar medicamentos conhecidos para o tratamento de doenças desconhecidas?

Apesar das dúvidas e das divergências de resultados nas pesquisas, o Ministério da Saúde liberou, para todos os estados brasileiros, o uso da HCLQ para pacientes em estado grave da COVID-19. Na nota técnica distribuida pelo governo (BRASIL, 2020)14 aparecem as seguintes razões:

2.1. Considerando a pandemia ocasionada pelo novo coronavirus humano (COVID-2019) declarada pela OMS e a situação epidemiológica brasileira (WHO, 2020a);

2.2. Considerando a inexistência de terapias farmacológicas e imunobiológicos especificos para COVID-19 e a taxa de letalidade da doença em individuos de idade avançada em razão da insuficiência de alternativas terapêuticas para essa população em especifico (BRASIL, 2020a);

2.3. Considerando as publicações recentes com dados preliminares sobre o uso da CLQ e HCLQ em pacientes com COVID-19 (Chatre, 2020, Touret, 2020; Gautret, 2020; Riera, 2020);

2.4. Considerando que o uso de CLQ é um tratamento de baixo custo, de fácil acesso e também facilmente administrada;

2.5. Considerando a capacidade nacional de produção de CLQ pelos laboratórios públicos brasileiros em larga escala e da capacidade de abastecimento desse medicamento a nivel estadual e municipal [...]

O Ministério da Saúde recomenda o uso apenas

\footnotetext{
13 BRASIL. Ministério da Saúde autoriza uso de cloroquina para casos graves de coronavírus. MS, 25 mar. 2020 . Disponivel em: https:// www.gov.br/pt-br/noticias/saude-e-vigilancia-sanitaria/2020/03/ministerio-da-saude-autoriza-uso-de-cloroquina-para-casos-graves-de-coronavirus. Acesso em: 30 abr. 2020

14 MINISTÉRIO DA SAÚDE. NOTA INFORMATIVA No 5/2020-DAF/SCTIE/MS. Disponivel em: https://ww/w.saude.gov.br/images/ pdf/2020/marco/30/MS---0014167392---Nota-Informativa.pdf. Acesso em: 30 abr. 2020.
} 
para pacientes graves. Mas as razões elencadas pelo Ministério da Saúde envolvem apenas questões de conveniência. O documento não faz nenhuma referência às razões bioéticas que tornariam aceitável o uso off label da CLQ e HCLQ em caráter excepcional.

O que causa mais espanto, porém, é o uso de referências a pesquisas que desaconselham explicitamente o uso terapêutico em larga escala. Por exemplo, o trabalho de Riera (2020), citado na nota técnica, tem como conclusão:

Com base nos achados nesta revisão sistemática rápida, a eficácia e a segurança da HCLQ e da CLQ em pacientes com COVID-19 é INCERTA e seu uso de rotina para esta situação NÃO pode recomendado (sic) até que os resultados dos estudos em andamento possam avaliar seus efeitos de modo apropriado..$^{15}$

Esse foi o documento ao qual o Ministério da Saúde teve acesso ao elaborar a Nota 5 mencionada acima. Agora, em versões mais atualizadas do referido estudo, a partir do dia 27/03, a conclusão do estudo de Riera foi ligeiramente modificada, passando a apresentar razões mais claras para restringir o uso. Eis as principais modificações:

Com base nos achados destes dois estudos, a eficácia e a segurança da HCLQ e da CLQ em pacientes com COVID-19 ainda é incerta e seu uso de rotina para esta situação não deveria ser recomendado até que os resultados dos estudos em andamento possam avaliar seus efeitos (beneficios e riscos) de modo apropriado". ${ }^{16}$

Finalmente, principios bioéticos foram usados de forma mais explicita para discutir a permissibilidade ou não do uso off label. Concordamos com a conclusão e a defenderemos a seguir.

A questão bioética fundamental que precisamos formular agora é a seguinte: em tempos de pandemia, estamos moralmente justificados a pular etapas de pesquisa, ou usar off label medicamentos conhecidos, testados e aprovados para outras doenças? Antes de respondê-la, cabe lembrar que uma pesquisa com seres humanos, que procura determinar se remédios ou vacinas podem ser efetivamente usados em larga escala, precisa estar embasada em sólidos principios bioéticos. No Brasil, a Resolução 466/2012, do Conselho Nacional de Saúde, estabelece quatro principios que devem ser aplicados para garantir a eticidade da pesquisa científica envolvendo seres humanos: a) respeito ao participante da pesquisa em sua dignidade e autonomia, reconhecendo sua vulnerabilidade, assegurando sua vontade de contribuir e permanecer, ou não, na pesquisa, por intermédio de manifestação expressa, livre e esclarecida; b) ponderação entre riscos e benefícios, tanto conhecidos como potenciais, individuais ou coletivos, comprometendo-se com o máximo de benefícios e o mínimo de danos e riscos; c) garantia de que danos previsiveis serão evitados; e d) relevância social da pesquisa, o que garante a igual consideração dos interesses envolvidos, não perdendo o sentido de sua destinação sócio-humanitária. ${ }^{17}$ Esses principios são usados nas decisões da CONEP e são prima facie válidos. Eles garantem a eticidade da pesquisa científica que embasa a produção de novos medicamentos ou usos off label e, eventualmente, de uma vacina para o COVID-19.

Para responder à pergunta bioética formulada acima, é preciso lembrar também que há orientações legais claras sobre as diversas fases da pesquisa clínica. No Brasil, a legislação exige que novos fármacos, medicamentos, vacinas ou testes diagnósticos sejam testados em quatro fases. ${ }^{18}$ Existe uma fase pré-clínica onde experimentos são feitos in vitro (em testes laboratoriais) e, posteriormente, in vivo (em animais não-humanos). Na fase clínica, a pesquisa deve ser controlada, randomizada e cega. Na fase I da pesquisa clínica, os testes são feitos em seres humanos, mas em um número pequeno de pessoas que concordam em participar do teste na qualidade de voluntários. Essas pesquisas procuram estabelecer a segurança do novo fármaco ou vacina. Em geral,

\footnotetext{
15 RIERA R., PACHECO, RL. HCLQ e CLQ para infecção por COVID-19. Revisão sistemática rápida. Disponivel em: https://oxfordbrazilebm $\mathrm{com} /$ wp-content/uploads/2020/03/RS_rapida_hidroxi_Cloroquina_COVID19.pdf. Acesso em: 30 abr. 2020.

16 RIERA R., PACHECO, RL. HCLQ e CLQ para infecção por COVID-19. Revisão sistemática rápida. Disponível em: https://oxfordbrazilebm. com/wp-content/uploads/2020/04/RS_rapida_hidroxicloroquina_COVID19_atualizacao_19_04_20.pdf Acesso em: 30 abr. 2020.

17 Disponivel em: https://conselho.saude.gov.br/resolucoes/2012/Reso466.pdf. Acesso em: 28 abr. 2020.

18 A legislação relevante pode ser encontrada aqui: http://portal.anvisa.gov.br/regulamentacao Acesso em: 25 mar. 2020.
} 
essa fase dura três anos. A fase II envolve um estudo terapêutico piloto que visa demonstrar principalmente a eficácia do medicamento, isto é, se ele serve para tratar a doença. Nessa fase, o número de pessoas que participa do teste também é pequeno, mas as pessoas, diferentemente do que ocorre na fase I, sofrem da condição patológica para a qual se busca um tratamento ou vacina. Na fase II se procura, por exemplo, estabelecer a relação entre dose-resposta com o objetivo de permitir estudos terapêuticos mais amplos incluindo de segurança (toxicidade). Esse período dura também aproximadamente três anos. É somente na fase III, então, que estudos são realizados em grandes e variados grupos de pacientes para determinar, basicamente, duas variáveis: a relação entre risco/beneficio a curto e longo prazos e o valor terapêutico relativo comparado com outras drogas. É na terceira fase que se exploram também caracteristicas especiais do novo medicamento ou vacina, por exemplo, relativamente a certas faixas etárias. A duração dessa fase é mais longa e pode chegara aproximadamente quatro anos. É somente após o cumprimento dessas fases que se pode solicitar o registro para a agência responsável pelo licenciamento de medicamentos. Nos Estados Unidos, essa agência é a Food and Drug Administration (FDA); no Brasil, cabe à ANVISA licenciar novos medicamentos. Finalmente, numa quarta (IV) e última fase, serão feitas pesquisas depois de comercializado o produto para descobrir novas reações adversas, novas indicações, novos métodos de administração etc.

Em condições normais, testes têm início in vitro, em culturas de células nos laboratórios e, posteriormente, in vivo, ou seja, em animais não-humanos. Essas etapas pré-clínicas foram dispensadas para a COVID-19, embora existam algumas evidências laboratoriais do efeito da CLQ no coronavírus que recomendam, entretanto, confirmação por estudos clínicos. ${ }^{19}$ Além disso, algumas pesquisas com animais não-humanos também já existiam, mas foram feitas para o surto da SARS-Cov igualmente causada por um tipo de coronavirus. A passagem de estudos laboratoriais em animais não humanos para testes clínicos é sempre um processo complexo. Por isso, as evidências pré-clínicas podem ser insuficientes. Muitas vezes, os resultados das pesquisas in vitro não se confirmam nos testes clínicos. Além disso, já era sabida a limitação do efeito da CLQ em outras doenças análogas, por exemplo, para a influenza. ${ }^{20}$ Dito de outro modo, a extrapolação do seu uso para a COVID-19 parece exigir novos testes clínicos rigorosos. Conhecendo já os efeitos colaterais do medicamento em pessoas sadias, ainda precisamos avaliar a segurança do uso da HCLQ em pessoas com COVID-19. Afinal, o I.T. (Índice de Toxicidade) de um medicamento pode variar de uma doença para a outra. Portanto, novos estudos clínicos (das fases II, III e IV) são necessários para saber se o medicamento é realmente eficaz, seguro e se não traz efeitos tóxicos indesejáveis em pessoas acometidas pela COVID-19.

Conforme vimos, o governo brasileiro decidiu, pulando algumas dessas etapas, recomendar a HCLQ, associada à Azitromicina, para pacientes acometidos da COVID-19. Em outros termos, o Ministério da Saúde recomendou o seu uso off label para casos graves. Ora, em situações de urgência, como a da pandemia atual, o uso para pacientes graves parece razoável se não houver alternativas terapêuticas. Em outros termos, um uso limitado, para pacientes em estado crítico, com o devido acompanhamento médico, pode ser feito e, segundo jargão médico conhecido, trata-se apenas de um uso compassivo.

Outras instituições e profissionais, todavia, logo ampliaram o seu uso. Como temos visto, a Prevent Senior iniciou vários testes antes mesmo da autorização da CONEP. ${ }^{21}$ Uma das razões alegadas

19 WANG, M. et al. Remdesivir and chloroquine effectively inhibit the recently emerged novel coronavirus (2019-nCoV) in vitro. Cell Research, v. 30, Londres, 2020. p. 269-271. Disponivel em: https://www.ncbi.nlm.nih.gov/pmc/articles/PMC7054408/Acesso em: 20 abr 2020. 20 PATON, N. et.al. Chloroquine for influenza prevention: a randomised,double-blind, placebo controlled trial. Lancet Infect Dis, 11, p. 67783. 2011. Disponivel em: https://www.ncbi.nlm.nih.gov/pubmed/21550310. Acesso em 30 mar. 2020.

${ }_{21}$ WATANABE, P. Estudo da Prevent Senior com cloroquina não tinha autorização; governo vê indicios de fraude. Estudo começou em 26 de março, mas só recebeu autorização da comissão de ética em 14 de abril. São Paulo, Folha de São Paulo. Publicado em 20 de abril de 2020. Disponivel em: https://www1.folha.uol.com.br/equilibrioesaude/2020/04/estudo-da-prevent-senior-com-cloroquina-nao-tinha-autorizacao-governo-ve-indicios-de-fraude.shtml. Acesso em: 27 maio 2020. 
para isso é que a HCLQ é um medicamento que já vem sendo comercializado há décadas e que seus efeitos já seriam conhecidos. Por conseguinte, em situações análogas, dadas a urgência de uma pandemia e a ausência de medicamentos especíicos, algumas pessoas sugerem que seria aceitável o uso desses medicamentos para a nova doença.

De fato, os efeitos, inclusive colaterais, da HCLQ já são bastante conhecidos. Esses efeitos, para a maioria dos pacientes, são controláveis e não são fatais. Por exemplo, ela pode causar alterações cardiovasculares, mas, para pacientes hospitalizados, principalmente em UTIs, esses efeitos adversos podem ser monitorados e até mesmo revertidos. Como veremos na próxima seção, entretanto, alguns defendem até mesmo o uso da HCLQ fora dessas condições e com acompanhamento via telemedicina.

Na presença de estudos observacionais apenas, não randomizados, sem grupo de controle, não há, entretanto, como garantir que a HCLQ seja o melhor o tratamento; muito menos, que ela traga mais beneficios que maleficios para pacientes em estado não crítico. O próprio Ministério da Saúde e a ANVISA, recentemente (07/04/20), reafirmaram não ter mudado a diretriz, ou seja, que somente seja usada HCLQ para pacientes graves.22 Pensamos que essa atitude é moralmente fundamentada. Ela também é justificável sob o ponto de vista científico, ou seja, se um remédio funciona, precisamos entender o mecanismo para, eventualmente, aperfeiçoá-lo e passar a prescrevê-lo com menos incertezas. As indicações inequivocas da HCLQ (ou contraindicações) precisam estar apoiadas por evidências fortes de danos ou benefícios. Por conseguinte, estudos clínicos mais cuidadosos ainda precisam ser concluidos.

\section{Aguardando o resultado das pesquisas}

A OMS lançou, em 20/3/2020, o projeto "Solidarity", que tem como objetivo realizar testes com as drogas mais promissoras no tratamento contra da COVID-19. Há quatro drogas sendo analisadas: (1) Remdesivir, utilizada no tratamento do ebola; (2) CLQ, utilizada, como vimos, para tratar a malária; (3) Ritonavir ou Lopinavir, que faz parte do coquetel de tratamento do HIV; (iv) Interferon-beta, uma molécula envolvida na regulação da inflamação corporal. ${ }^{23}$ No Brasil, a Fiocruz coordenará a pesquisa que será aplicada para pacientes graves em 18 hospitais de 12 estados, com o apoio do Departamento de Ciência e Tecnologia (DECIT) do Ministério da Saúde. ${ }^{24} \mathrm{~A}$ própria OMS, portanto, também está fazendo testes de vários medicamentos durante a pandemia. Parece justificado, então, numa situação de calamidade pública, na presença de um novo virus, sem tratamento conhecido, sem vacinas e sem que nossos corpos estejam preparados com os devidos anticorpos etc. que pesquisas científicas sejam feitas sem seguir todas as etapas experimentais normais. Essa atitude é, em tempos de pandemia, uma atitude eticamente justificável.

Mesmo que seja, todavia, adequado pular etapas de pesquisa ou fazer uso off label restrito e acompanhado por profissionais/pesquisadores competentes, não parece moralmente correto indicar o uso indiscriminado de um medicamento sem que haja conhecimento necessário para tal. Propomos, dessa maneira, prestar mais atenção aos princípios bioéticos da pesquisa com seres humanos nos estudos clínicos em curso e ao juramento Hipocrático no uso off label nas práticas clínicas ou usar até mesmo o princípio da precaução para se evitar danos maiores aos participantes das pesquisas e/ou aos pacientes. ${ }^{25}$

\footnotetext{
22 SALDANA, P., MACHADO, R. Enquanto Mandetta reitera cuidado com cloroquina, Bolsonaro volta a defender remédio contra coronavirus. Folha de S. Paulo, 7 abr. 2020. Disponivel em: https://www1.folha.uol.com.br/equilibrioesaude/2020/04/enquanto-mandetta-reitera-cuidado-com-cloroquina-bolsonaro-volta-a-defender-remedio-contra-coronavirus.shtml Acesso em: 8 maio 2020.

${ }_{23}$ Mais informações podem ser obtidas na página da OMS, disponivel em: https://www.who.int/emergencies/diseases/novel-coronavirus-2019/global-research-on-novel-coronavirus-2019-ncov Acesso em: 31 abr. 2020.

${ }_{24}$ Detalhes podem ser obtidos na própria página da Fundação, disponível em: https://portal.fiocruz.br/noticia/fiocruz-lidera-no-brasil-ensaio-clinico-solidarity-solidariedade-da-oms. Acesso em: 8 abr. 2020.

25 Cf. COMEST - World Commission on the Ethics of Science and Technology. The precautionary principle. Paris: UNESCO, 2005, p. 14.
} 
Quais seriam os danos em questão? Pesquisas científicas mostram que a CLQ é bastante tóxica e tem vários efeitos negativos, alguns dos quais já foram mencionados acima. Uma lista mais completa, baseada na bula do Difosfato de Cloroquina inclui: efeitos cardiovasculares (hipotensão, vasodilatação, supressão da função miocárdica, arritmias cardiacas, parada cardiaca) e do sistema nervoso (confusão, convulsões e coma); no tratamento oral podem causar cefaleia, irritação do trato gastrointestinal, distúrbios visuais e urticária; doses diárias altas (>250 mg), em doses cumulativas de mais de $1 \mathrm{~g} / \mathrm{Kg}$ de CLQ base, podem resultar em retinopatia e ototoxicidade irreversiveis; tratamento prolongado com altas doses também pode causar miopatia tóxica, cardiopatia e neuropatia periférica, visão borrada, diplopia, confusão, convulsões, erupções, quineloides na pele, embranquecimento dos cabelos, alargamento do complexo QRS e anormalidade da onda $\mathrm{T}$; em casos raros podem ocorrer hemólise e discrasias sanguíneas. A HCLQ é menos tóxica, mas não deixa de ter efeitos colaterais. Se, agora, contrastarmos com os potenciais beneficios, quase inexistentes contra o novo coronavirus, então poderemos decidir a partir de princípios bioéticos a permissibilidade de seu uso ou não. Poucos benefícios são apontados nos estudos que defendem o uso da CLQ e, conforme vimos na primeira seção deste artigo, há sérias dúvidas quanto ao estatuto científico das conclusões. Por conseguinte, quando os malefícios superam os benefícios, profissionais da saúde estão sob a obrigação de suspender tratamentos ou nada fazer (primum non nocere).

Por um lado, o uso compassivo da HCLQ pode ser feito, pois, no ambiente hospitalar, é possivel controlar os efeitos e tomar as medidas necessárias para diminuir os danos. Por outro lado, deve-se evitar o uso indiscriminado. Nesse sentido, a recomendação restritiva do Ministério da Saúde parece adequada e segue orientações iniciais do Conselho Federal de Medicina (CFM), da própria OMS e pode ser interpretada como uma aplicação de princípios sólidos. É temerário ampliar indevidamente o uso de medicamentos experimentais, pois prometeremos um suposto tratamento a pacientes sem ter evidências científicas suficientes dos seus efeitos. Dado que não há razões suficientes, não se deve ministrar HCLQ como remédio capaz de curar ou mesmo tratar todos os sintomas da COVID-19.

Mais importante ainda sob o ponto de vista bioético, é que precisamos testar outras drogas para saber se não temos alternativas melhores. Por exemplo, os testes clínicos com Remdesivir ou até mesmo Avigan já estão bastante adiantados. Um artigo recente publicado na prestigiosa revista Journal of the American Medical Association (JAMA), mostra que, até o momento, o Remdesivir parece ter as melhores perspectivas terapêuticas. ${ }^{26}$ Existem, hoje, mais de 200 ensaios clínicos em andamento registrados no clinicaltrials.gov, incluindo alguns estudos brasileiros que usam a CLQ, por exemplo, no Hospital Pronto-Socorro Zephelina Aziz, em Manaus, com o seguinte identificador NCT04323527. ${ }^{27}$ A insistência no uso da CLQ ou da HCLQ pode ser desastroso. Por exemplo, experimento amazonense consiste em tratar diferentes grupos de pacientes com diferentes doses de CLQ: para alguns, 600 mg/dia durante 10 dias. No terceiro dia, esses pacientes começaram a apresentar arritmia e 11 deles foram a óbito, o que levou à suspensão deste ramo da pesquisa. ${ }^{28}$ Recordando os princípios bioéticos citados acima, cabe perguntar: os pacientes sabiam que estavam correndo altíssimos riscos com poucas perspectivas de benefícios? Eles assinaram um Termo de Consentimento Livre e Esclarecido ou apenas uma simples "anuência"?

\footnotetext{
26 SANDERS, J. et al. Pharmacologic Treatments for Coronavirus Disease 2019 (COVID-19)

A Review. Journal of the American Medical Association. doi:10.1001/jama.2020.6019. Disponivel em: https://jamanetwork.com/journals/ jama/fullarticle/2764727. Acesso em: 14 abr. 2020.

27 U. S. NATIONAL LIBRARY OF MEDICINE. Disponivel em: https://www.clinicaltrials.gov/ct2/show/NCT04323527?term=Ncto4323527\&draw $=2 \&$ rank $=1$. Acesso em: 14 abr. 2020.

28 THOMAS, K. SHEIKH, K. Small Chloroquine Study Halted Over Risk of Fatal Heart Complications. 12 abr. 2020. Disponivel em: https:// www.nytimes.com/2020/04/12/health/chloroquine-coronavirus-trump.html?smid=fb-share\&fbclid=IwARodYEw_k6fBAwSQZwVfbU4k2_hs2jPjMEz7HvQdUwokywd_XokxOKqQpG8. Acesso em: 14 abr. 2020.
} 
Afinal, trata-se de pesquisas com seres humanos que devem ser respeitados em sua autonomia. Essas perguntas também devem ser endereçadas às outras instituições que estão fazendo estudos similares. Aliás, também a Prevent Senior anunciou um experimento em larga escala. Pelo que foi divulgado à imprensa, o estudo já teria demonstrado a cura de 300, de um total de 500 pacientes, em estágio inicial da COVID-19, com o uso da HCLQ com Azitromicina e será publicado em breve. ${ }^{29}$ Cabe perguntar se o estudo é randomizado, se foi estabelecido algum grupo de controle, ou seja, se segue uma metodologia científica rigorosa. O estudo, entretanto, parece contradizer outras pesquisas e atitudes adotadas internacionalmente de suspensão de tais tratamentos. ${ }^{30}$ A Prevent Senior vai mais longe: valendo-se da recente aprovação da telemedicina, prescreve e entrega na casa do paciente não hospitalizado, os medicamentos para uso off label. ${ }^{31} \mathrm{~A}$ hipótese é a de que a HCLQ, se administrada precocemente em pacientes ambulatoriais com COVID-19 moderada, impediria a replicação do coronavírus, reduzindo com isso a morbidade da doença (isto é, as complicações que resultam da forma grave da doença, a qual exige hospitalização). ${ }^{32}$ Porém, o desenho metodológico do estudo é repleto de falhas. No site da Livraria Nacional de Medicina do Instituto Nacional de Saúde (NIH) dos Estados Unidos, o estudo foi descrito (corretamente) por seus autores como um estudo "exploratório". Ora, estudos exploratórios não têm o objetivo de provar hipóteses, mas aventá-las. Com um estudo desse tido, nenhuma conclusão é capaz de garantir que é o "tratamento" que está promovendo a cura da doença nessas fases iniciais (do terceiro ao quinto dia dos primeiros sintomas) e não o próprio organismo do paciente, reagindo e vencendo o vírus com seus anticorpos. Como é sabido, de um ponto de vista científico, correlação não implica causalidade.

Enfim, e infelizmente, como, aliás, destaca o editorial do JAMA, em 13 de abril de 2020: Nenhuma terapia mostrou-se efetiva até então. Dada essa situação atual de inexistência de estudos completos e na ausência de medicamentos específicos ou vacinas para a COVID-19, nada pode ser afirmado conclusivamente.

Sem os resultados de estudos clínicos bem delineados, não há como saber qual é o melhor remédio off label disponivel para combater a COVID-19. Alegar, como fazem algumas autoridades brasileiras, que a CLQ é mais barata e menos prejudicial à economia do que priorizar o isolamento social como estratégia não é colocar a vida e o bem-estar das pessoas como prioridade. Pode-se estar repetindo, hoje, o mesmo equívoco cometido há alguns anos quando, de forma apressada e sem evidências suficientes, foi aprovado o uso da fosfoetanolamina sintética (conhecida como "pílula azul") para tratamento de câncer. ${ }^{33}$ Por conseguinte, sustentamos que o uso off label da HCLQ deveria, excepcionalmente em tempos de pandemia, ser feito apenas nos termos descritos na nota técnica do Ministério da Saúde, evitando-se, assim, seu uso em larga escala.

Para finalizar, então, endossamos aqui a mensagem da International Society of Drug Bulletins sobre a busca de medicamentos para a COVID-19: "os ensaios randomizados são a única forma de compilar informação de qualidade sobre como tratar a COVID-19. Os medicamentos experimentais como Cloroquina e Hidroxicloroquina somente devem ser usados no marco de um bom ensaio

29 DESIDÉRIO, M. O Estudo da Prevent Senior que já curou 300 pessoas. São Paulo, Exame, 8 abr. 2020. Disponível em: https://exame. abril.com.br/negocios/tudo-o-que-se-sabe-sobre-a-pesquisa-da-prevent-senior-com-cloroquina/Acesso em 14 abr. 2020

3o WALLIN. C. Hospitais de ponta da Suécia interromperam o uso do medicamento cloroquina em pacientes infectados com o coronavírus, em consequência de relatos de graves efeitos colaterais - como arritmias cardiacas e perda de visão periférica. 10 abr. 2020 Disponivel em: https://noticias.uol.com.br/ultimas-noticias/rfi/2020/04/10/hospitais-da-suecia-suspendem-uso-de-cloroquina-em-pacientes-com-coronavirus-devido-a-efeitos-colaterais.htm Acesso em: 14 abr. 2020.

31 MACHADO, L. Prevent Senior contraria recomendação e receita medicamento a pacientes que não estão internados com coronavirus. 4 abr. 2020. Disponivel em: https://g1.globo.com/sp/sao-paulo/noticia/2020/04/03/prevent-senior-contraria-recomendacao-do-ministerio-e-receita-medicamento-a-pacientes-sem-confirmacao-de-coronavirus.ghtml. Acesso em 30 abr. 2020.

32 U. S. NATIONAL LIBRARY OF MEDICINE. Disponivel em: https://clinicaltrials.gov/ct2/show/NCT04348474 Acesso em: 19 abr. 2020.

33 LEDFORD, H. Brazilian law grants patients right to use untested cancer 'drug'. But researchers warn that there is no real evidence that it actually works. Nature, 15 abr. 2016. Disponivel em: https://www.nature.com/news/brazilian-law-grants-patients-right-to-use-untested-cancer-drug-1.19756. Acesso em: 30 abr. 2020. 
clínico, com um protocolo rigoroso e aderindo-se de forma estrita ao método de compilação de dados"34 (grifo nosso). Dito de outro modo, enquanto não tivermos os resultados científicos dos ensaios clínicos, é melhor seguir os princípios bioéticos. Nesse sentido, o recente Parecer 04/2020 do CFM, que "autoriza", mas não "recomenda", o uso da HCLQ, também não foi oportuno e poderia ter esperado o resultado das pesquisas. ${ }^{35}$

O que vem ocorrendo atualmente, portanto, é um processo rápido de testagem clínica. Não é admissivel que haja uma liberação para que todos os pacientes recebam esse medicamento sem o devido acompanhamento hospitalar e médico. Nossa proposta, então, é que sejam observados os estudos e testes relevantes e que somente após a confirmação clínica proporcionada por estudos metodologicamente bem delineados, tais como os que estão sendo conduzidos pela Coalizão COVID Brasil e pela Solidarity, seja liberado e recomendado um uso mais amplo de medicamentos como a HCLQ.

\section{Considerações finais}

Os estudos científicos apontam para a falta de evidências conclusivas do valor terapêutico do uso da HCLQ, associada ou não à azitromicina, para tratar a COVID-19. Vimos, no presente trabalho, que estamos justificados, em casos excepcionais de pandemia e na ausência de medicamentos conhecidos, a pular etapas nos ensaios clínicos. Bioeticamente falando, todavia, isso somente pode ser feito na medida que temos algumas indicações claras de que estamos produzindo algum benefício ao paciente. Concluímos, então, que a restrição do Ministério da Saúde no uso da HCLQ teve embasamento ético e científico.

Se essa conclusão acima estiver correta, então é precipitada a liberação do uso da HCLQ para todos os casos. É recomendável, moral e cientificamente, esperar os resultados das pesquisas que estão sendo feitas pela Coalizão COVID Brasil e pela Solidarity para uso em larga escala. Se quisermos proporcionar um cuidado realmente respeitoso aos pacientes, precisamos seguir os padrões éticos das pesquisas cientíicas e ensaios clínicos, principalmente, para usos off label de medicamentos.

Diante de uma grave crise, devemos redobrar o rigor ético, e não o relaxar. Em tempos de pandemia, as pessoas, principalmente aquelas acometidas por uma doença desconhecida, que naturalmente causa medo e insegurança, estão vivenciando um estado de maior vulnerabilidade. Sempre coube à Bioética, preocupada com os aspectos normativos da pesquisa cientíica, protegê-las. Portanto, em situações de emergência, capazes de produzir grandes calamidades, a Bioética é mais necessária do que nunca, pois as decisões que tomamos afetam milhares de vidas. Todos nós queremos a cura por medicamentos e, preferencialmente, uma vacina para a COVID-10. Mas, neste momento, tudo o que nós temos a prescrever sem contraindicações é um bom procedimento preventivo, a saber, a quarentena e o distanciamento social. Esta atitude não apenas ajuda a "achatar a curva" e aliviar o SUS (Sistema Único de Saúde) como também dá tempo aos profissionais de saúde e pesquisadores para testarem clinicamente remédios mais eficazes (mesmo off label) e seguros, aos cientistas para desenvolverem medicamentos especíicos para a COVID-19 e quiçá, em alguns poucos meses, uma vacina.

\section{REFERÊNCIAS}

AZEVEDO, Marco. The Precautionary Principle. In: DOMINGUES, I. Biotechnologies and the Human Condition. Belo Horizonte: Editora UFMG, 2012.

BEAUCHAMP, T.; CHILDRESS, J. Principles of Biomedical Ethics. 7. ed. Oxford: Oxford University Press, 2016.

DALL'AGNOL. Bioética. 2. ed. Rio de Janeiro: Zahar, 2015.

DOMINGUES, I. Biotechnologies and the Human Condition. Belo Horizonte: Editora UFMG, 2012.

\section{Alcino Eduardo Bonella}

Professor na Universidade Federal de Uberlândia (UFU) em Uberlândia, MS, Brasil. Pesquisador do CNPq. 


\section{Marcelo de Araujo}

Doutor em Filosofia pela Universidade de Konstanz (Alemanha). Professor Associado de Ética da Universidade do Estado do Rio de Janeiro (UERJ) no Rio de Janeiro, RJ, Brasil e professor Associado IV de Filosofia do Direito da Universidade Federal do Rio de Janeiro (UFRJ), Rio de Janeiro, RJ, Brasil. Pesquisador do CNPq.

\section{Darlei Dall'Agnol}

Doutor em Filosofia na University of Bristol. Professor titular da Universidade Federal de Santa Catarina (UFSC) em Florianópolis, SC, Brasil, Pesquisador do CNPq.

\section{Endereço para correspondência}

Alcino Eduardo Bonella

Av. dos Vinhedos, 900

38411040

Uberlândia, MG, Brasil

Marcelo de Araujo

Rua Prof. Luiz Cantenhede, 77 ap.403

Laranjeiras, 22245-040

Rio de Janeiro, RJ, Brasil

Darlei Dall'Agnol

Universidade Federal de Santa Catarina

Campus Universitário Trindade

Caixa Postal 476

88040970

Florianópolis, SC, Brasil 